Review Article

\title{
Neurologic and musculoskeletal effects of tilt-table standing on adults: a systematic review
}

\author{
RichaRd W. BohannON $\left.{ }^{1}\right)^{*}$, MiChelle D GREen $^{2)}$ \\ 1) Physical Therapy Consultants: Fuquay-Varina, NC, USA \\ 2) Department of Physical Therapy, Campbell University, USA
}

\begin{abstract}
Purpose] Tilt table use is associated, most often, with the assessment of syncope. However, it also has applications for patients with neurologic and orthopedic problems. These applications do not appear to be widely applied. The purpose of this review, therefore, was to summarize the research literature addressing the use of tilt tables for treating specific musculoskeletal and neurologic impairments in adults. [Methods] Relevant literature was identified by searches of the PubMed, CINAHL, and Scopus databases and hand searches (December 2018 and October 2020). The methodological quality of the identified research articles was assessed using the PEDro scale. [Results] Of 482 unique articles identified, 20 matched the eligibility criteria of the review and were included. The studies varied widely in the populations studied, procedures used, and responses reported. The studies provide limited support for tilt table standing as an intervention. [Conclusion] However, evidence that some patients with neurologic conditions may respond positively to tilt-table standing is available. Among such individuals are those with decreased ankle range of motion, positive neurologic signs in the lower limbs, and decreased levels of consciousness.

Key words: Tilt-table, Range of motion, Spasticity
\end{abstract}

(This article was submitted May 4, 2021, and was accepted Jun. 27, 2021)

\section{INTRODUCTION}

The Guide to Physical Therapist Practice describes one component of the physical therapist management of patients as the "prescription, application, and, as appropriate, fabrication or modification" of assistive technology- of which tilt-tables can be considered an example ${ }^{1}$. A survey of physical therapists working in intensive care units in Australia indicated that a majority used tilt-tables ${ }^{2}$. An observational study of patients with stroke demonstrated that most of those treated on tilt-tables tolerated the procedure well ${ }^{3}$. These facts notwithstanding, we have not observed widespread therapeutic use of tilt-tables in clinical settings in the United States- perhaps because of the cost of tilt-tables, the need to monitor hemodynamic status during tilt-table standing, or the perception that tilt-table standing is a passive activity. The purpose of this systematic review, therefore, was to determine whether tilt-table standing is justified on the basis of neurologic or musculoskeletal effects accompanying the intervention.

\section{METHODS}

Articles addressing the use of tilt-table standing to achieve neurologic or musculoskeletal benefits for adults were identified by an electronic search of 3 databases (PubMed, CINAHL, Scopus) on December 20, 2018 and again on October 15, 2020. The electronic searches used the search string "Tilt-table AND (Physical Therapy OR Rehabilitation)". A hand-search was also conducted. To be included an article had to report neurologic or musculoskeletal effects of standing with assistance

*Corresponding author. Richard W. Bohannon (E-mail: rbohannonpt@gmail.com)

(C2021 The Society of Physical Therapy Science. Published by IPEC Inc.

(c) (i) $\odot$ This is an open-access article distributed under the terms of the Creative Commons Attribution Non-Commercial No DerivaCC BY NC ND tives (by-nc-nd) License. (CC-BY-NC-ND 4.0: https://creativecommons.org/licenses/by-nc-nd/4.0/) 
of a conventional tilt-table. Articles reporting use of a tilt-table in combination with other interventions were included if tilttable standing was judged to be a fundamental component of the intervention. Articles focused on use of robotic tilt-tables, standers or tilting beds were excluded. Articles were also excluded if written in a language other than English.

The 2 authors independently reviewed potentially relevant articles identified by the computerized and hand searches. They initially selected articles based on their titles and abstracts. When the titles or abstracts provided insufficient information to guide selection, the full text of articles was consulted. Included articles were perused by the authors for information about study participants, interventions, and neurologic and musculoskeletal effects of the interventions. The PEDRO scale was used to grade the quality of the included studies ${ }^{4}$.

\section{RESULTS}

Three hundred forty-four unique articles were identified by the computerized searches. Twenty-two additional articles were found using hand searches (Fig. 1). Ultimately 20 articles were identified as relevant on the basis of inclusion and exclusion criteria $^{5-24)}$. The articles are summarized in Table 1 . Research originated from 11 countries. The number of neurologically or musculoskeletally involved individuals treated on a standard tilt-table ranged from 1 to 38. Although individuals with stroke or brain injury were the most commonly studied, individuals with other nervous system problems (eg, spinal cord injury) were also investigated. Responses to a single session or multiple sessions were studied. Tilt-table standing sessions were sometime limited by orthostatic intolerance ${ }^{15,19)}$, but sessions typically involved standing times of 20 to 40 minutes and table angles of $70^{\circ}$ or more from horizontal. In 8 studies participants stood on a wedge or incline plates ${ }^{6,7,9,14,16-18,23)}$.

The effect examined most consistently in the included articles was ankle dorsiflexion range of motion. Two studies reported ankle range of motion to increase over the course of a single standing session ${ }^{17,23)}$. Of studies focused on changes in ankle range of motion over multiple standing sessions, 4 reported a small increase in ankle dorsiflexion range of motion $9,14,20,21$ ) and 1 documented a small decrease ${ }^{7)}$. Notably, however, the decrease was less in weightbearing limbs undergoing stretching than in non-weightbearing limbs not undergoing stretching ${ }^{7}$. Closely related to range of motion is ankle stiffness. Two studies showed resistance to passive ankle dorsiflexion to decrease significantly over the course of a tilt-table session ${ }^{17}$, 18) Odéen and Knutsson reported the decreases to be greater in patients who stood on the tilt table with the ankle dorsiflexed rather than plantarflexed ${ }^{18)}$.

The effects of tilt-table standing on positive motor signs varied. Of 6 studies grading spasticity using the Ashworth or Tardieu scales $5,8,14,15,19,23), 2$ noted an improvement (albeit not significant) after individual sessions of tilt-table standing ${ }^{8,23)}$. One of the 2 studies also produced improved performance on the pendulum test ${ }^{8}$. Bohannon, as well as Adams and Hicks, reported an improvement in extensor spasms after tilt-table standing ${ }^{5,8)}$, but Adams and Hicks did not note improvement in flexor spasms or clonus after tilt-table standing ${ }^{5)}$. Two investigative groups have described favorable changes in motor neuron excitability after a session of tilt-table standing, but only the changes reported by Tsai et al were significant ${ }^{6,23)}$.

A broad array of other responses to tilt-table standing have been documented. Chief among these is the state of arousal and consciousness in patients with altered consciousness. Riberholt et al observed among patients with brain injury that tilt-table standing was associated with a significantly increased time with eyes open ${ }^{19}$. Krewer et al. reported significant increases in

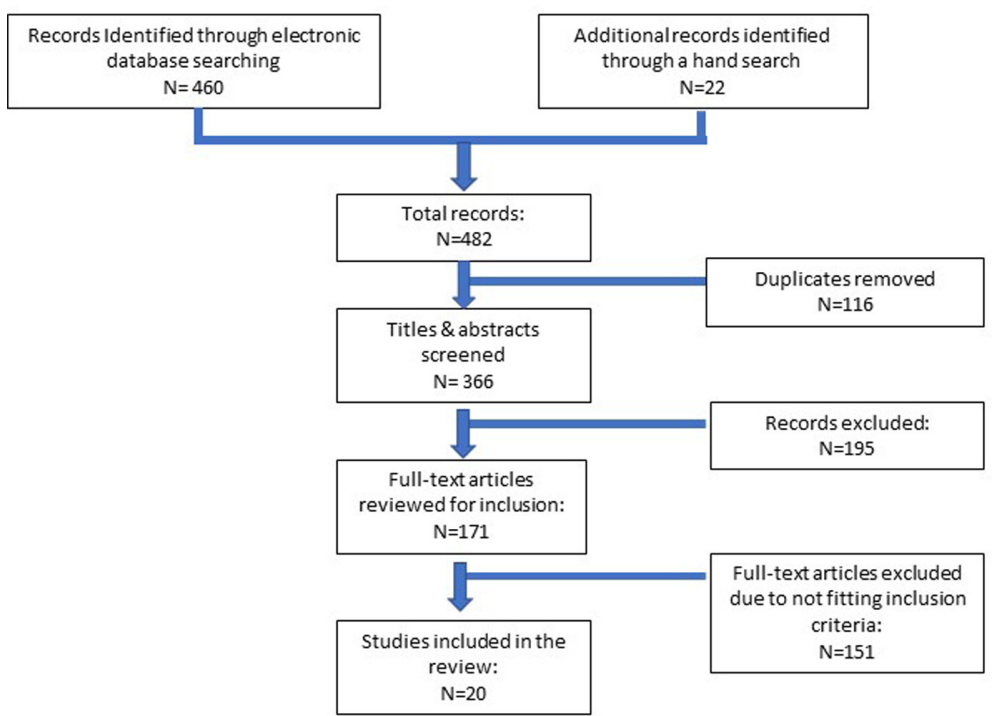

Fig. 1. Study flow diagram according to preferred reporting items for systematic reviews and meta-analyses (PRISMA). 
Table 1. Summary of 20 articles included in systematic review

\begin{tabular}{|c|c|c|c|}
\hline Study & Population & Intervention & Findings \\
\hline $\begin{array}{l}\text { Adams \& } \\
\text { Hicks }^{5}\end{array}$ & $\begin{array}{l}\text { Canadians with } \\
\text { chronic spinal cord } \\
\text { injury }(\mathrm{n}=7)\end{array}$ & $\begin{array}{l}\text { TT angle: greatest tolerated encouraged } \\
\text { (maximum } 80^{\circ} \text {, mean } 68.6^{\circ} \text { ). } \\
\text { Time: } 45 \text { min maximum during } 12 \text { sessions } \\
\text { over } 4 \mathrm{wk} \\
\text { Standing: bilaterally } \\
\text { Other: body-weight supported treadmill } \\
\text { training performed over another } 4 \mathrm{wk}\end{array}$ & $\begin{array}{l}\text { TT resulted in notable } 0.9 \text { point } \downarrow \text { in extensor } \\
\text { spasm severity after a single session \& } 1.0 \text { point } \\
\downarrow \text { after } 4 \text { wk. No favorable } \Delta \text { in MAS scores, } \\
\text { flexor spasm severity, clonus severity, spasm } \\
\text { frequency, spasticity impact, quality of life, or } \\
\text { function. }\end{array}$ \\
\hline $\begin{array}{l}\text { Bakheit, } \\
\text { et al. }{ }^{6)}\end{array}$ & $\begin{array}{l}\text { English with stroke \& } \\
\text { spasticity ( } \mathrm{n}=66 ; 22 / \\
\text { group) \& healthy con- } \\
\text { trols ( } \mathrm{n}=21 ; 7 / \text { group) }\end{array}$ & $\begin{array}{l}\text { TT angle: "vertical". } \\
\text { Time: } 20 \text { min during } 1 \text { session. } \\
\text { Standing: on footplate adjusted to maximum } \\
\text { ankle dorsiflexion. } \\
\text { Other: isokinetic or isotonic stretch. }\end{array}$ & $\begin{array}{l}\text { Excitability of ankle plantarflexors as indicated } \\
\text { by } \mathrm{H}_{\max }: \mathrm{M}_{\max } \& \mathrm{H} \text {-reflex latency } \downarrow \text { but not } \\
\text { SGNF post-stretch \& } 24 \text { h post-stretch in TT or } \\
\text { other groups. }\end{array}$ \\
\hline $\begin{array}{l}\text { Ben, } \\
\text { et al. }\end{array}$ & $\begin{array}{l}\text { Australians with re- } \\
\text { cent spinal cord injury } \\
(\mathrm{n}=20)\end{array}$ & $\begin{array}{l}\text { TT angle: max tolerated, vertical encour- } \\
\text { aged } \\
\text { Time: } 30 \mathrm{~min}, 3 \times / \mathrm{wk} \text { for } 20 \mathrm{wk} \text {. } \\
\text { Standing: } 1 \text { lower limb on block and } 15^{\circ} \\
\text { wedge. } \\
\text { Other: control limb unsupported (non- } \\
\text { weightbearing) }\end{array}$ & $\begin{array}{l}\text { Ankle dorsiflexion } \downarrow \text { a mean } 1^{\circ} \text { in weightbear- } \\
\text { ing limb } \& \downarrow 5^{\circ} \text { in nonweightbearing limb. Total } \\
\text { proximal bone mineral density } \downarrow 0.06 \mathrm{~g} / \mathrm{cm}^{2} \text { in } \\
\text { the weightbearing and nonweightbearing limbs. }\end{array}$ \\
\hline Bohannon $^{8)}$ & $\begin{array}{l}\text { American with } \\
\text { chronic spinal cord } \\
\text { injury }(\mathrm{n}=1)\end{array}$ & $\begin{array}{l}\text { TT angle: } 80^{\circ} \\
\text { Time: } 30 \text { min on } 5 \text { nonconsecutive days } \\
\text { Standing: bilaterally. } \\
\text { Other: not stated }\end{array}$ & $\begin{array}{l}\text { Spasms \& spasticity of knee extensors as docu- } \\
\text { mented by observation, pendulum test \& MAS } \\
\text { scores } \downarrow \& \text { transfers improved immediately after } \\
\text { intervention but returned to baseline within } 24 \\
\text { hours. }\end{array}$ \\
\hline $\begin{array}{l}\text { Bohannon, et } \\
\text { al. }{ }^{9)}\end{array}$ & $\begin{array}{l}\text { Americans with } \\
\text { assorted neurologic } \\
\text { disorders: mostly } \\
\text { stroke }(\mathrm{n}=20)\end{array}$ & $\begin{array}{l}\text { TT angle: } 70^{\circ} \\
\text { Time: } 30 \text { min on } 5-22 \text { days } \\
\text { Standing: bilaterally on wedges } \\
\text { Other: individually tailored treatments. }\end{array}$ & $\begin{array}{l}\text { Mean passive ankle dorsiflexion } \mathrm{ROM} \uparrow 8^{\circ} \\
\text { (range } 3^{\circ} \text { to } 17^{\circ} \text { ) over course of intervention. }\end{array}$ \\
\hline $\begin{array}{l}\text { Elliot, } \\
\text { et al. }{ }^{10)}\end{array}$ & $\begin{array}{l}\text { English with brain } \\
\text { injury } \\
(\mathrm{n}=12)\end{array}$ & $\begin{array}{l}\text { TT angle: } 85^{\circ} \\
\text { Time: } 20 \text { min repeated over } 1 \mathrm{wk} \\
\text { Other: lying in bed }\end{array}$ & $\begin{array}{l}\text { Highest rank \& total number of behaviors of } \\
\text { Wessex Head Injury Matrix during standing } \\
\text { SGNF }>\text { during lying in bed. }\end{array}$ \\
\hline $\begin{array}{l}\text { Kim, } \\
\text { et al. }{ }^{11)}\end{array}$ & $\begin{array}{l}\text { Korean's with stroke } \\
(\mathrm{n}=34 ; 11-12 / \text { group })\end{array}$ & $\begin{array}{l}\text { TT angle: comfortable } \\
\text { Time: } 20 \text { min/day for } 6 \text { wk. } \\
\text { Standing: bilaterally (group 1), on more af- } \\
\text { fected paretic lower limb (groups } 2 \& 3 \text { ) } \\
\text { Other: Group } 2 \text { rhythmically flexed and } \\
\text { extended less affected lower limb. Group } \\
3 \text { performed task specific training of less } \\
\text { affected upper limb. All groups received } 30 \\
\text { min routine therapy. }\end{array}$ & $\begin{array}{l}\text { Group 1: SGNF } \uparrow \text { in paretic Fugl-Meyer up- } \\
\text { per limb score ( } 3.4 \text { points) but not Wolf Motor } \\
\text { Function Test score }(0.9 \text { points) or grip strength. } \\
\text { ( } 0.9 \mathrm{~kg}) \text {. } \\
\text { Group } 2 \text { : SGNF } \uparrow \text { in paretic Fugl-Meyer upper } \\
\text { limb score }(9.2 \text { points) \& grip strength }(4.7 \mathrm{~kg}) \\
\text { but not Wolf Motor Function Test score }(0.8 \\
\text { points) } \\
\text { Group 3: SGNF } \uparrow \text { in paretic Fugl-Meyer upper } \\
\text { limb score ( } 10.5 \text { points), Wolf Motor Function } \\
\text { Test score ( } 10.1 \text { points), \& grip strength }(13.1 \mathrm{~kg}) \text {. }\end{array}$ \\
\hline $\begin{array}{l}\text { Kim, } \\
\text { et al. }{ }^{12)}\end{array}$ & $\begin{array}{l}\text { Korean's with stroke } \\
(\mathrm{n}=34,11-12 / \text { group })\end{array}$ & $\begin{array}{l}\text { TT angle: Comfortable to maximum of } 90^{\circ} \text {. } \\
\text { Time: } 20 \text { min/day, } 5 \text { days/wk for } 3 \text { wk. } \\
\text { Standing: bilaterally (group 2), on more } \\
\text { affected paretic limb (group 3). Group } 3 \\
\text { performed task oriented training. } \\
\text { Other: All groups (including group } 1 \text { [con- } \\
\text { trol]) received } 30 \text { min routine therapy. }\end{array}$ & $\begin{array}{l}\text { Group } 2: \text { SGNF } \uparrow \text { in Barthel Index score }(53.4 \\
\text { points), } \downarrow \text { in National Institutes of Health Stroke } \\
\text { Scale (score } 4.9 \text { points), } \uparrow \text { in Fugl-Meyer score } \\
\text { (11.9 points). } \\
\text { Group 3: SGNF } \uparrow \text { in Barthel Index score ( } 59.9 \\
\text { points), } \downarrow \text { in score } 5.6 \text { points), } \uparrow \text { in Fugl-Meyer } \\
\text { score ( } 17.3 \text { points). }\end{array}$ \\
\hline $\begin{array}{l}\text { Krewer, } \\
\text { et al. }{ }^{13)}\end{array}$ & $\begin{array}{l}\text { Germans with severe } \\
\text { disorders of con- } \\
\text { sciousness ( } \mathrm{n}=38,19 / \\
\text { group) }\end{array}$ & $\begin{array}{l}\text { TT angle: } 70^{\circ} \\
\text { Time: } 30 \mathrm{~min}, 3-4 \text { sessions/wk for } 3 \mathrm{wk} \text {. } \\
\text { Standing: not specified } \\
\text { Other: Erigo TT with robotic stepping de- } \\
\text { vice. therapist selected additional therapy. }\end{array}$ & $\begin{array}{l}\text { Coma Recovery Scale scores } \uparrow \text { SGNF from me- } \\
\text { dian } 12 \text { points @ baseline, to median } 17 \text { points } \\
\text { @ } 3 \text { wk, to median } 19 \text { points @ } 6 \text { wk. }\end{array}$ \\
\hline
\end{tabular}


Table 1. Continued

\begin{tabular}{|c|c|c|c|}
\hline Study & Population & Intervention & Findings \\
\hline $\begin{array}{l}\text { Leung, } \\
\text { et al. }{ }^{14)}\end{array}$ & $\begin{array}{l}\text { Australians with } \\
\text { traumatic brain injury } \\
(\mathrm{n}=15)\end{array}$ & $\begin{array}{l}\text { TT angle: as upright as tolerated } \\
\text { Time: } 30 \text { min, } 3 \times / \text { wk for } 6 \text { wk, then } \\
4 \times / \text { wk } \\
\text { Standing: bilaterally without wedge. } \\
\text { Other: Experimental group supplemented } \\
\text { TT standing with wedges \& electrical stimu- } \\
\text { lation to ankle dorsiflexors \& splinting. }\end{array}$ & $\begin{array}{l}\text { Mean passive ankle dorsiflexion ROM with } 12 \\
\text { Nm stretching force } \uparrow 3^{\circ} \text { from }-6^{\circ} \text { (baseline) to } \\
-3^{\circ}(6 \& 10 \mathrm{wk}) \text {. } \\
\text { Mean spasticity score (Tardieu) } \uparrow \text { from } 1 \text { (base- } \\
\text { line) to } 3 \text { ( } 6 \mathrm{wk}) \& 2 \text { ( } 10 \mathrm{wk} \text { ). } \\
\text { Mean gait speed } \uparrow \text { from } 0.1 \mathrm{~m} / \mathrm{s} \text { (baseline) to } 0.4 \\
\mathrm{~m} / \mathrm{s}(6 \& 10 \mathrm{wk} \text { ) } \\
\text { Mean FIM gait score } \uparrow \text { from } 2 \text { (baseline) to } 3 \text { (6 } \\
\& 10 \mathrm{wk} \text { ) }\end{array}$ \\
\hline $\begin{array}{l}\text { Luther, } \\
\text { et al. }{ }^{15)}\end{array}$ & $\begin{array}{l}\text { Germans with sub- } \\
\text { acute brain injury } \\
\text { who were comatose or } \\
\text { semicomatose }(n=9)\end{array}$ & $\begin{array}{l}\text { TT angle: incrementally to } 70^{\circ} \text {. } \\
\text { Time: min not stated, } 1 \text { day } \\
\text { Other: TT with stepping device }\end{array}$ & $\begin{array}{l}\text { No SGNF } \uparrow \text { in state of consciousness as mea- } \\
\text { sured by Coma Recovery Scale-Revised score } \\
\text { during standing. Spasticity as measured by the } \\
\text { MAS did not } \downarrow \text { SGNF with standing. }\end{array}$ \\
\hline $\begin{array}{l}\text { Maynard, } \\
\text { et al. }{ }^{16)}\end{array}$ & $\begin{array}{l}\text { English with chronic } \\
\text { stroke ( } \mathrm{n}=66 ; 22 / \\
\text { group) \& healthy con- } \\
\text { trols ( } \mathrm{n}=21 ; 7 / \text { group) }\end{array}$ & $\begin{array}{l}\text { TT angle: "vertical". } \\
\text { Time: } 20 \text { min during } 1 \text { session. } \\
\text { Standing: on footplate adjusted to maximum } \\
\text { ankle dorsiflexion. } \\
\text { Other: isokinetic or isotonic stretch }\end{array}$ & $\begin{array}{l}\text { No SGNF improvement in any measured kine- } \\
\text { matic, kinetic, or spatio-temporal gait parameter } \\
\text { immediately or } 24 \mathrm{~h} \text { after standing. }\end{array}$ \\
\hline $\begin{array}{l}\text { Ochi, } \\
\text { et al. }{ }^{17)}\end{array}$ & $\begin{array}{l}\text { Japanese with sub- } \\
\text { acute stroke }(n=9)\end{array}$ & $\begin{array}{l}\text { TT angle: not stated } \\
\text { Time: } 10 \mathrm{~min}, 2 \text { days/wk, for } 2 \mathrm{wk} \text {. } \\
\text { Standing: on incline plates. } \\
\text { Other: Condition 1: no arm cranking. Condi- } \\
\text { tion 2: arm cranking. Routine rehabilitation. }\end{array}$ & $\begin{array}{l}\text { Ankle dorsiflexion ROM } \uparrow \text { SGNF by } 8.9 \% \& \\
11.7 \% \text { on condition } 1 \text { days } \& 8.9 \% \& 14.0 \% \text { on } \\
\text { condition } 2 \text { days. } \\
\text { Ankle dorsiflexion stiffness } \downarrow \text { SGNF by } 8.0 \% \& \text { } \\
7.9 \% \text { on condition } 1 \text { days } \& 20.7 \% \& 24.2 \% \text { on } \\
\text { condition } 2 \text { days. } \\
\text { Gait velocity did not } \triangle \text { SGNF on condition } 1 \text { or } \\
2 \text { days. }\end{array}$ \\
\hline $\begin{array}{l}\text { Odéen \& } \\
\text { Knutsson }^{18)}\end{array}$ & $\begin{array}{l}\text { Swedes with chronic } \\
\text { spinal cord injury } \\
(\mathrm{n}=9)\end{array}$ & $\begin{array}{l}\text { TT angle: } 85^{\circ} \\
\text { Time: } 30 \text { min, } 4 \text { days } \\
\text { Standing: with ankles dorsiflexed or plan- } \\
\text { tarflexed } 10^{\circ}-15^{\circ} \\
\text { Other: Ankle braced in dorsiflexion while } \\
\text { patient supine }\end{array}$ & $\begin{array}{l}\text { Mean resistance to ankle dorsiflexion at } 0.25 \\
\text { cycle } / \mathrm{s} \downarrow 15 \% \text { (SGNF) in TT dorsiflexion condi- } \\
\text { tion and } 11 \% \text { in TT plantarflexion condition. } \\
\text { Mean resistance to ankle dorsiflexion at } 1.0 \\
\text { cycle } / \mathrm{s} \downarrow 32 \% \text { (SGNF) in TT dorsiflexion condi- } \\
\text { tion and } 26 \% \text { (SGNF) in TT plantarflexion } \\
\text { condition. }\end{array}$ \\
\hline $\begin{array}{l}\text { Riberholt, et } \\
\text { al. }{ }^{19)}\end{array}$ & $\begin{array}{l}\text { Danes with subacute } \\
\text { aquired brain injury } \\
(\mathrm{n}=16)\end{array}$ & $\begin{array}{l}\text { TT angle } 80^{\circ} \\
\text { Time: } 20 \text { min target during } 1 \text { session. } \\
\text { Standing: no stipulation } \\
\text { Other: Standard rehabilitation. }\end{array}$ & $\begin{array}{l}\text { Time }(66 \%) \text { with eyes open during intervention } \\
\text { SGNF }>\text { time }(22.1 \%) \text { before intervention. MAS } \\
\text { was not SGNF different before \& after interven- } \\
\text { tion }\end{array}$ \\
\hline Richardson $^{20)}$ & $\begin{array}{l}\text { English with subacute } \\
\text { head injury }(\mathrm{n}=1)\end{array}$ & $\begin{array}{l}\text { TT angle: not stated } \\
\text { Time: patient tolerance, } 7 \text { days } \\
\text { Standing: less affected lower limb on box, } \\
\text { more involved limb on footplate of TT table. } \\
\text { Other: Standard rehabilitation. }\end{array}$ & $\begin{array}{l}\text { Ankle dorsiflexion ROM during intervention } \\
\left.\text { period (mean }-16^{\circ}\right)<\text { during preintervention } \\
\text { baseline }\left(\text { mean }-26.7^{\circ}\right)\end{array}$ \\
\hline $\begin{array}{l}\text { Robinson, } \\
\text { et al. }{ }^{21)}\end{array}$ & $\begin{array}{l}\text { Australians with acute } \\
\text { stroke }(n=13)\end{array}$ & $\begin{array}{l}\text { TT angle: not stated } \\
\text { Time: } 30-40 \mathrm{~min}, 5 \text { days/wk, for } 4 \mathrm{wk} \\
\text { Standing: less affected lower limb on box, } \\
\text { more involved limb on footplate of TT. } \\
\text { Other: night splint group. All received inpa- } \\
\text { tient rehabilitation }\end{array}$ & $\begin{array}{l}\text { Ankle dorsiflexion } \mathrm{ROM} \uparrow 0.8^{\circ} \text { after } 4 \mathrm{wk} \& \\
5.9^{\circ} \text { after } 10 \mathrm{wk} \text {. Standing up ability (Motor As- } \\
\text { sessment Scale) } \uparrow \text { mean } 1.4 \text { points after } 4 \mathrm{wk} \& \\
2.2 \text { points after } 10 \mathrm{wk} .\end{array}$ \\
\hline $\begin{array}{l}\text { Toccolini, } \\
\text { et al. }{ }^{22)}\end{array}$ & $\begin{array}{l}\text { Brazilians in intensive } \\
\text { care units }(\mathrm{n}=23)\end{array}$ & $\begin{array}{l}\text { TT angle: progressively increased to } 90^{\circ} \text { as } \\
\text { tolerated. } \\
\text { Time: } 30 \text { min as tolerated during multiple } \\
\text { days. } \\
\text { Standing: not specified }\end{array}$ & $\begin{array}{l}\text { During tilting } \uparrow \text { noted in Glasgow Coma Scale \& } \\
\text { Richmond Agitation-Sedation scale depending } \\
\text { on tilt angle \& session. }\end{array}$ \\
\hline
\end{tabular}


Table 1. Continued

\begin{tabular}{|c|c|c|c|}
\hline Study & Population & Intervention & Findings \\
\hline $\begin{array}{l}\text { Tsai, } \\
\text { et al. }{ }^{23)}\end{array}$ & $\begin{array}{l}\text { Taiwanese with } \\
\text { chronic stroke }(n=17)\end{array}$ & $\begin{array}{l}\text { TT angle: } 85^{\circ} \\
\text { Time: } 30 \text { min, } 1 \text { day } \\
\text { Standing: ankles maximally dorsiflexed } \\
\text { using wedges } \\
\text { Other: Standard rehabilitation }\end{array}$ & $\begin{array}{l}\text { Passive ankle dorsiflexion } \mathrm{ROM} \uparrow \mathrm{SGNF} \text { (mean } \\
5.1^{\circ} \text { ) between pretreatment } \& \text { posttreatment. } \\
\text { MAS scores did not } \Delta \text { SGNF. Motor neuron } \\
\text { excitability (H/M ratio) } \downarrow \text { SGNF }(31.8 \% \text { ) after } \\
\text { intervention; excitability (F/M) ratio } \uparrow \text { SGNF } \\
(118 \% \text { ) after intervention). MAS scores for ankle } \\
\text { plantarflexor muscles } \downarrow \text { but not SGNF after } \\
\text { treatment }\end{array}$ \\
\hline $\begin{array}{l}\text { Wilson, } \\
\text { et al. }{ }^{24)}\end{array}$ & $\begin{array}{l}\text { English in vegetive or } \\
\text { minimally conscious } \\
\text { state }(n=16)\end{array}$ & $\begin{array}{l}\text { TT: } 90^{\circ} \text { as tolerated. } \\
\text { Time: once }\end{array}$ & $\begin{array}{l}\text { Number of behaviors of Wessex Head Injury } \\
\text { Matrix during standing SGNF > during lying } \\
\text { supine }\end{array}$ \\
\hline
\end{tabular}

@: at; $\Delta$ : change; $\downarrow$ : decrease; $\uparrow:$ increase; MAS: Modified Ashworth Scale; min: minutes; ROM: range of motion; SGNF: significant or significantly; TT: tilt table; wk: week.

Table 2. Quality rating of 20 articles according to PEDro scale

\begin{tabular}{lcccccccccccc}
\hline Reference & Item 1 & Item 2 & Item 3 & Item 4 & Item 5 & Item 6 & Item 7 & Item 8 & Item 9 & Item 10 & Item 11 & Sum \\
\hline 5 & 1 & 1 & 0 & 1 & 0 & 0 & 0 & 1 & 1 & 1 & 1 & 7 \\
6 & 1 & 1 & 1 & 1 & 0 & 0 & 0 & 1 & 1 & 1 & 1 & 8 \\
7 & 1 & 1 & 1 & 1 & 0 & 0 & 1 & 1 & 1 & 1 & 1 & 9 \\
8 & 1 & 0 & 0 & 0 & 0 & 0 & 0 & 1 & 0 & 0 & 0 & 2 \\
9 & 1 & 0 & 0 & 0 & 0 & 0 & 0 & 1 & 1 & 0 & 1 & 4 \\
10 & 0 & 0 & 0 & 0 & 0 & 0 & 0 & 1 & 1 & 1 & 1 & 4 \\
11 & 1 & 1 & 1 & 1 & 0 & 0 & 1 & 1 & 1 & 1 & 1 & 9 \\
12 & 1 & 1 & 1 & 1 & 0 & 0 & 1 & 1 & 1 & 1 & 1 & 9 \\
13 & 1 & 1 & 0 & 1 & 0 & 0 & 0 & 0 & 1 & 1 & 1 & 6 \\
14 & 1 & 1 & 1 & 1 & 0 & 0 & 1 & 1 & 1 & 1 & 1 & 9 \\
15 & 1 & 1 & 1 & 1 & 0 & 0 & 0 & 1 & 1 & 1 & 1 & 8 \\
16 & 1 & 1 & 1 & 1 & 0 & 0 & 0 & 1 & 1 & 1 & 1 & 8 \\
17 & 1 & 1 & 0 & 1 & 0 & 0 & 0 & 0 & 1 & 1 & 1 & 6 \\
18 & 1 & 0 & 0 & 0 & 0 & 0 & 0 & 1 & 1 & 1 & 1 & 5 \\
19 & 1 & 0 & 0 & 0 & 0 & 0 & 0 & 1 & 1 & 1 & 1 & 5 \\
20 & 0 & 0 & 0 & 0 & 0 & 0 & 0 & 0 & 1 & 0 & 1 & 2 \\
21 & 1 & 1 & 1 & 1 & 0 & 0 & 1 & 0 & 1 & 1 & 1 & 8 \\
22 & 1 & 0 & 0 & 0 & 0 & 0 & 0 & 0 & 1 & 1 & 1 & 4 \\
23 & 1 & 0 & 0 & 0 & 0 & 0 & 0 & 1 & 1 & 1 & 1 & 5 \\
24 & 0 & 0 & 0 & 0 & 0 & 0 & 0 & 1 & 1 & 1 & 1 & 4 \\
\hline
\end{tabular}

PEDRO items: 1. Eligibility criteria specified, 2. Participants randomly allocated to groups, 3. Allocation concealed, 4. Groups similar at baseline, 5. Participants blinded, 6. Therapy providers blinded, 7. Assessors blinded, 8. Key outcome obtained from $>85 \%$ of allocated participants, 9. All participants received treatment or control condition as allocated, 10. Results of between-group comparison reported for at least one outcome, 11 . Point and variability measures reported. Scoring: $0=$ no, $1=$ yes.

Coma Recovery Scale scores over a 3-week regimen of standing in patients in a vegetative or minimally conscious state ${ }^{13)}$. Toccolini et al. found standing to be associated with improvements in the Glasgow Coma Scale and Richmond Agitation -Sedation scale scores ${ }^{22}$. Elliot et al. and Wilson et al. reported positive behavioral responses with standing according to the Wessex Head Injury Matrix ${ }^{10,24)}$. Luther et al did not find consistent improvement in Coma Recovery Scale scores with standing ${ }^{15}$. Among other responses to tilt table standing Ben et al found bone mineral density to decrease similarly in weightbearing and non-weightbearing limbs of patients with spinal cord injury in spite of their participation in a 20-week regimen of tilt-table standing 7 ). Motor recovery, as reflected in various index scores (eg, Fugl-Meyer, Barthel, and NIH Stroke Scale), has been demonstrated (albeit not consistently) to improve following tilt-table standing ${ }^{11,12)}$. Although not consistently or significantly, specific activities such as walking ${ }^{14,16,17)}$, transferring ${ }^{8)}$, and standing up ${ }^{21)}$ have been shown to improve in some studies.

Table 2 summarizes the quality ratings of the reviewed articles. The scores ranged from 2 to 9 out of 11 . The median score 
was 7. Notably, no study met the blinding criteria for participants or therapy providers. All but 1 study specified eligibility criteria. Only 1 study failed to provide point and variability measures.

\section{DISCUSSION}

This systematic review was undertaken to determine if studies focused on neurologic or musculoskeletal effects of tilttable standing support its use as a therapeutic intervention for adults. Relevant studies and sample sizes were limited in number, involved only patients with neurologic conditions, were diverse in regard to procedures, and demonstrated inconsistent findings.

The strongest evidence for tilt-table standing appears to be its association with small increases in ankle dorsiflexion range of motion over the course of a session or multiple sessions. The one study not finding such an increase did find the loss of range of motion to be less on the weightbearing limb. The use of a wedge may increase the effectiveness of tilt-table standing by reducing the plantarflexors' excitability and resistance to stretch. Notwithstanding this reduction in excitability and reports of some dramatic results in one case, evidence that tilt-table standing consistently results in reduced spasticity and spasms is mixed. We, like NG and King ${ }^{25}$, conclude that studies addressing the effects of tilt-table standing on arousal are mostly positive but equivocal. Findings relative to standing and improvements in motor recovery and function are equivocal. The combination of purposive motor tasks with tilt-table standing may be augmentary. We found no evidence that tilt-table standing has a favorable effect on bone-mineral density.

Considering the findings and quality of the studies reviewed herein, an unqualified endorsement of tilt-table standing does not seem warranted. That noted, some individuals with neurologic disorders do appear to be responders to the intervention. Tilt-table standing therefore may be worth a trial with patients with specific problems. This is particularly the case if the tilttable allows a therapeutic intervention (eg, prolonged high-load stretching of the ankle plantarflexor muscles) not otherwise practicable.

Further research into the value of tilt-table standing is clearly warranted. Such research could assist in the identification of patient and interventional variables affecting response to tilt-table standing. Among patient variables, degree of spasticity and time since onset might be important. Duration of standing might be a germane interventional variable if the intent is to affect ankle range of motion- particularly as no study we reviewed involved more than 40 minutes of standing per session and Tardieu et al have described 2 hours of stretching per day as inadequate to prevent progressive contracture ${ }^{26}$.

\section{Conflict of interest}

None.

\section{REFERENCES}

1) Assistive Technology: Prescription, Application, and, as Appropriate, Fabrication or Modification. Guide to Physical Therapist Practice. http://guidetoptpractice.apta.org/content/1/SC34.body (Accessed Mar. 1, 2019)

2) Chang AT, Boots R, Hodges PW, et al.: Standing with assistance of a tilt table in intensive care: a survey of Australian physiotherapy practice. Aust J Physiother, 2004, 50: 51-54. [Medline] [CrossRef]

3) Baltz MJ, Lietz HL, Sausser IT, et al.: Tolerance of a standing tilt table protocol by patients an inpatient stroke unit setting: a pilot study. J Neurol Phys Ther, 2013, 37: 9-13. [Medline] [CrossRef]

4) de Morton NA: The PEDro scale is a valid measure of the methodological quality of clinical trials: a demographic study. Aust J Physiother, 2009, 55: 129-133. [Medline] [CrossRef]

5) Adams MM, Hicks AL: Comparison of the effects of body-weight-supported treadmill training and tilt-table standing on spasticity in individuals with chronic spinal cord injury. J Spinal Cord Med, 2011, 34: 488-494. [Medline] [CrossRef]

6) Bakheit AM, Maynard V, Shaw S: The effects of isotonic and isokinetic muscle stretch on the excitability of the spinal alpha motor neurones in patients with muscle spasticity. Eur J Neurol, 2005, 12: 719-724. [Medline] [CrossRef]

7) Ben M, Harvey L, Denis S, et al.: Does 12 weeks of regular standing prevent loss of ankle mobility and bone mineral density in people with recent spinal cord injuries? Aust J Physiother, 2005, 51: 251-256. [Medline] [CrossRef]

8) Bohannon RW: Tilt table standing for reducing spasticity after spinal cord injury. Arch Phys Med Rehabil, 1993, 74: 1121-1122. [Medline] [CrossRef]

9) Bohannon RW, Larkin PA: Passive ankle dorsiflexion increases in patients after a regimen of tilt table-wedge board standing. A clinical report. Phys Ther, 1985, 65: 1676-1678. [Medline] [CrossRef]

10) Elliott L, Coleman M, Shiel A, et al.: Effect of posture on levels of arousal and awareness in vegetative and minimally conscious state patients: a preliminary investigation. J Neurol Neurosurg Psychiatry, 2005, 76: 298-299. [Medline] [CrossRef]

11) Kim CY, Kim HD: Effect of crossed-education using a tilt table task-oriented approach in subjects with post-stroke hemiplegia: a randomized controlled trial. J Rehabil Med, 2018, 50: 792-799. [Medline] [CrossRef]

12) Kim CY, Lee JS, Kim HD, et al.: Lower extremity muscle activation and function in progressive task-oriented training on the supplementary tilt table during stepping-like movements in patients with acute stroke hemiparesis. J Electromyogr Kinesiol, 2015, 25: 522-530. [Medline] [CrossRef]

13) Krewer C, Luther M, Koenig E, et al.: Tilt table therapies for patients with severe disorders of consciousness: a randomized, controlled trial. PLoS One, 2015, 10: e0143180. [Medline] [CrossRef] 
14) Leung J, Harvey LA, Moseley AM, et al.: Standing with electrical stimulation and splinting is no better than standing alone for management of ankle plantarflexion contractures in people with traumatic brain injury: a randomised trial. J Physiother, 2014, 60: 201-208. [Medline] [CrossRef]

15) Luther MS, Krewer C, Müller F, et al.: Comparison of orthostatic reactions of patients still unconscious within the first three months of brain injury on a tilt table with and without integrated stepping. A prospective, randomized crossover pilot trial. Clin Rehabil, 2008, 22: 1034-1041. [Medline] [CrossRef]

16) Maynard V, Bakheit AM, Shaw S: Comparison of the impact of a single session of isokinetic or isotonic muscle stretch on gait in patients with spastic hemiparesis. Clin Rehabil, 2005, 19: 146-154. [Medline] [CrossRef]

17) Ochi A, Fukumoto M, Takami R, et al.: Effect of ankle stretching combined with arm cycling on the improvement of calf muscle stiffness in patients with stroke: a pilot study. J Phys Ther Sci, 2018, 30: 1305-1309. [Medline] [CrossRef]

18) Odéen I, Knutsson E: Evaluation of the effects of muscle stretch and weight load in patients with spastic paraplegia. Scand J Rehabil Med, 1981, 13: 117-121. [Medline]

19) Riberholt CG, Thorlund JB, Mehlsen J, et al.: Patients with severe acquired brain injury show increased arousal in tilt-table training. Dan Med J, 2013, 60: A4739. [Medline]

20) Richardson DL: The use of the tilt-table to effect passive tendo-Achillis stretch in a patient with head injury. Physiother Theory Pract, 1991, 7: 45-50. [CrossRef]

21) Robinson W, Smith R, Aung O, et al.: No difference between wearing a night splint and standing on a tilt table in preventing ankle contracture early after stroke: a randomised trial. Aust J Physiother, 2008, 54: 33-38. [Medline] [CrossRef]

22) Toccolini BF, Osaku EF, de Macedo Costa CR, et al.: Passive orthostatism (tilt table) in critical patients: clinicophysiologic evaluation. J Crit Care, 2015, 30: 655.e1-655.e6. [Medline] [CrossRef]

23) Tsai KH, Yeh CY, Chang HY, et al.: Effects of a single session of prolonged muscle stretch on spastic muscle of stroke patients. Proc Natl Sci Counc Repub China B, 2001, 25: 76-81. [Medline]

24) Wilson BA, Dhamapurkar S, Tunnard C, et al.: The effect of positioning on the level of arousal and awareness in patients in the vegetative state or the minimally conscious state: a replication and extension of a previous finding. Brain Impair, 2013, 14: 475-479. [CrossRef]

25) Ng H, King A: A systematic review of head-up tilt to improve consciousness in people with a prolonged disorder of consciousness. Clin Rehabil, 2021, 35: 13-25. [Medline] [CrossRef]

26) Tardieu C, Lespargot A, Tabary C, et al.: For how long must the soleus muscle be stretched each day to prevent contracture? Dev Med Child Neurol, 1988, 30: 3-10. [Medline] [CrossRef] 